\title{
A Novel Y-Suture Transfixation Technique to Improve Graft Adherence in High-Risk Descemets Stripping Automated Endothelial Keratoplasty
}

\author{
John Cason1,2,5, Habeeb Ahmad1,2, Pho Nguyen"1,2,3, Martin Heur1,2, Jonathan Song1.2, \\ Samuel C. Yiu ${ }^{1,2,4^{*}}$ \\ ${ }^{1}$ Doheny Eye Institute, Los Angeles, CA, USA \\ ${ }^{2}$ Department of Ophthalmology, Keck School of Medicine of the University of Southern California, Los Angeles, \\ CA, USA \\ ${ }^{3}$ Southern California Permanente Physician Group, Riverside, CA, USA \\ ${ }^{4}$ Department of Ophthalmology, The Wilmer Eye Institute, The John Hopkins University, Baltimore, Maryland, \\ USA \\ ${ }^{5}$ Department of Ophthalmology, Naval Medical Center San Diego, San Diego, USA \\ Email: syiu2@jhmi.edu
}

Received 15 June 2014; revised 22 July 2014; accepted 23 August 2014

Copyright (C) 2014 by authors and Scientific Research Publishing Inc.

This work is licensed under the Creative Commons Attribution International License (CC BY).

http://creativecommons.org/licenses/by/4.0/

c) (i) Open Access

\section{Abstract}

Purpose: To report a novel Y-suture transfixation technique to improve success rate of high-risk Descemets stripping automated endothelial keratoplasties (DSAEK). Design: Retrospective noncomparative case series. Participants: Twenty five high-risk patients undergoing DSAEK at one tertiary care institution. Methods: A retrospective evaluation of consecutively enrolled high-risk DSAEK cases performed by three surgeons at one institution during a 3-year period (2007-2010). Main outcome measures were graft adherence and suture-related complications. Results: Highrisk factors were defined as followed: prior incisional glaucoma surgery $(58 \%)$, presence of vitreous in the anterior chamber (15\%), anterior chamber intraocular lens $(8 \%)$, graft dislocation $(8 \%)$, previous DSAEK (8\%), and iris trauma/aphakia (3\%). Twenty-five high-risk patients underwent DSAEK with Y-suture transfixation. Postoperative follow-up ranged from 3 to 27 months. One patient had dislocation due to hypotony from prior trabeculectomy $(4 \%)$, requiring repeat DSAEK. Neither primary graft failure nor suture-related complication was observed. Conclusion: The Y-suture transfixation technique is associated with improved success rate of high-risk DSAEK transplant.

\footnotetext{
${ }^{*}$ Corresponding author.
} 


\section{Keywords}

\section{Descemets Stripping Automated Endothelial Keratoplasty, Dislocated Graft Prevention, Sutured Lenticule}

\section{Introduction}

Endothelial keratoplasty (EK) has become the preferred surgical remedy for corneal endothelial diseases. The Eye Bank Association of America reported that numbers of corneal tissue provided for EK procedures increased by 1287\% from 1429 in 2005 to 18,375 in 2008 [1]. The success of EK, particularly Descemet stripping with automated endothelial keratoplasty (DSAEK), is largely due to its remarkably expeditious visual rehabilitation and favorable complication rates compared to penetrating keratoplasty (PKP) [2]-[6]. Among the repertoire of EK procedures, DSAEK emerges as the procedure of choice for the corneal surgeons. [7] Derived from posterior lamellar keratoplasty pioneered by Melles [8] [9] and later modified by Terry [3] [10] and others, DSAEK has revolutionized the treatment of corneal endothelial disease. The technique has evolved over the past decade with respect to tissue handling and technique of tissue insertion. However, postoperative complications are not uncommon.

Graft detachment, either a partial or complete loss of adherence between the donor lenticule and host stroma, is a relatively common postoperative occurrence and can lead to deleterious complications [5] [11]-[12]. Lee and colleagues reported a graft detachment rates from $0 \%$ to $82 \%$ in most studies, with an average of $14.5 \%$ [5]. Predisposition factors include hypotony, iris trauma, glaucoma drainage tubes, trabeculectomy blebs, aphakia, vitreous in the anterior chamber, and prior graft detachment. Accordingly, to reduce the risks of graft detachment, we present a novel method, named the Y-suture transfixation, which can be of use in those who are at risk.

\section{Method}

Patients: A total of 25 DSAEK surgeries were performed on 25 patients in a non-consecutive fashion at the Doheny Eye Institute in Los Angeles by three different surgeons (J.S., S.Y., M.H). All surgeries were performed from 2007 through 2010. Inclusion criteria consisted of corneal edema in those associated with prior failed DSAEK, prior glaucoma surgery (blebs and tubes), hypotony, aphakia, and the presence of vitreous in the anterior chamber. The Institutional Review Board at the University of Southern California approved the study protocol.

Surgical Technique: Donor lenticules were prepared via independent eye banks, precut for the surgeons, and stored in Optisol GS (Optisol GS [Bausch \& Lomb Surgical, Irvine, CA]). Donor free-cap lenticule diameters ranged from 9 to10 $\mathrm{mm}$, and the thickness of the lenticules ranged from 100 to $150 \mu \mathrm{m}$.

After a partial conjunctival peritomy and dissection, a 5.2-mm scleral tunnel incision was constructed. A caliper was then used to mark $8 \mathrm{~mm}$ on the cornea to aid in the Descemetorhexis. Two angled paracentesis incisions were also made superiorly and inferiorly. Cohesive ophthalmic viscoelastic device (OVD) was injected into the anterior chamber to maintain spacing and pressure in the eye. Scoring of the Descemets membrane was achieved using a reverse Sinskey hook. A Rosenwasser stripper was then used to remove the Descemets membrane. Aggressive scraping of the peripheral border of the Descemetorhexis was performed to promote better adherence of the lenticule, as described by Terry [3]. The OVD was then removed by irrigation and aspiration.

After trephination using 8.0-mm trephines, a small amount of cohesive OVD was placed in the center of the donor lenticule. The lenticule was then folded in a 40/60-taco configuration. Using Charlie forceps, the folded lenticule was inserted through the 5.2-mm scleral wound into the anterior chamber. The scleral tunnel was closed with 10-0 nylon sutures. The anterior chamber was expanded carefully with balanced salt solution injected through the paracentesis incision on the side of the fold. An air bubble was then injected to unfold the lenticule and to promote adherence of the donor lenticule to the host stroma. A massage cannula was used to center the lenticule and to squeegee out residual interface fluid. A period of surgical rest ranging from 10 to 20 minutes was used to promote adherence of the lenticule. Afterwards, three 10.0 nylon sutures on a TG160 needle were used to place three peripheral anchoring sutures in a tangential fashion, securing the donor lenticule to the host cornea. The sutures were 120 degrees apart to create a "Y-formation." The knots were rotated peri- 
pherally in the superficial cornea but left exposed to minimize tissue trauma (Figure 1, Figure 2). Subconjunctival cefazolin and dexamethasone were administered and the eyelid speculum was removed. The patient remained in the postoperative recovery unit for 1 hour in a face-up and supine posture. The patient was encouraged to maintain that posture as much as possible during the next 24-hour period. Postoperative medications included a fourth-generation fluoroquinolone and prednisolone acetate four times each day. A bandage contact lens was placed over the cornea on the first postoperative day visit. The sutures were typically removed 1 week later, once the absence of graft-interface fluid was verified.

\section{Results}

Retrospective analysis was performed on a total of 25 patients (12 males, 13 females) who underwent 25 consecutive DSAEK surgeries using the Y suture transfixation technique. Fifteen patients (60\%) had prior glaucoma surgery, with either a trabeculectomy or a aqueous tube shunt. The vitreous in the anterior chamber was observed in four cases (16\%). Prevalence of risk factors are illustrated in Figure 2.

Postoperative follow-up ranged from 3 to 27 months. The primary endpoints were graft detachment and suture-related complication (s). Ninety-six percent of the DSAEK surgeries in these high-risk patients demonstrated full adherence. During postoperative recovery, there was one lenticule detachment in a patient with evident bleb leakage (4\%), shown on anterior segment optical coherence tomography (Figure 3). After this leak was repaired, the patient underwent an unremarkable repeat DSAEK; however, the second DSAEK was excluded from analyses. All 24/25 grafts were clear at the last follow-up visit; there were no graft rejections, primary graft failures, or suture-related complications in the series (Figure 4).

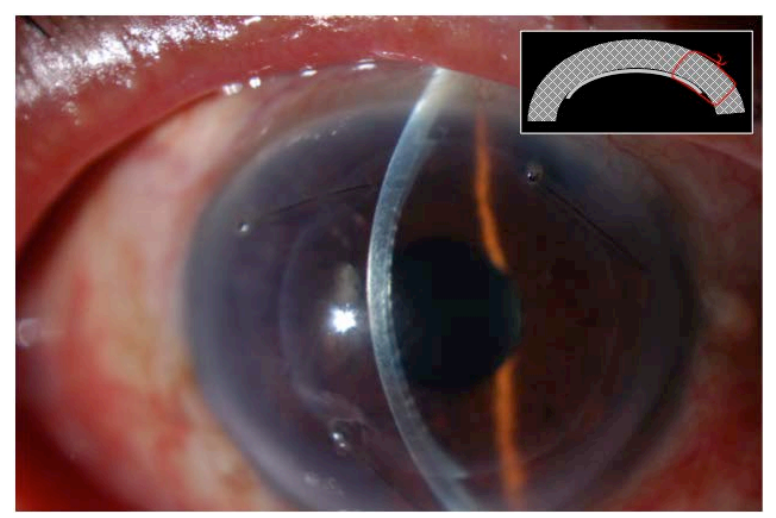

Figure 1. Color photograph of a patient at postoperative week 1 . Three interrupted 10-0 nylon sutures placed through the full-thickness cornea including both host and donor cornea (inset). These sutures are placed 120 degrees apart and slanted to minimize trauma to the donor graft. Note the three full-thickness sutures and tight adherence of the donor lenticule despite the presence of a glaucoma drainage device at 10 -clock hour.

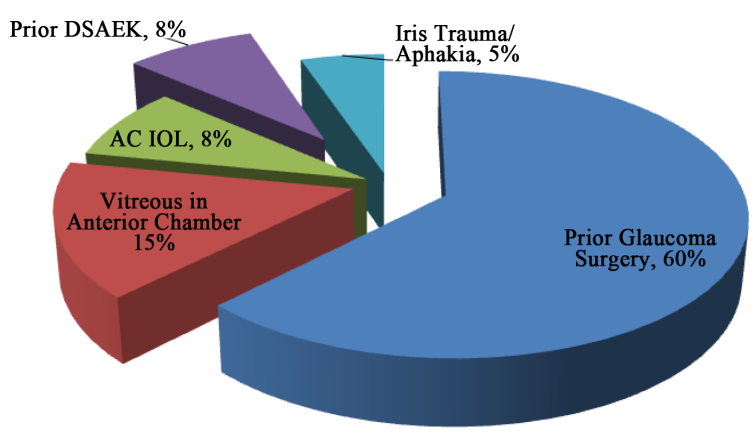

Figure 2. Indications for Y-suture transfixation. 


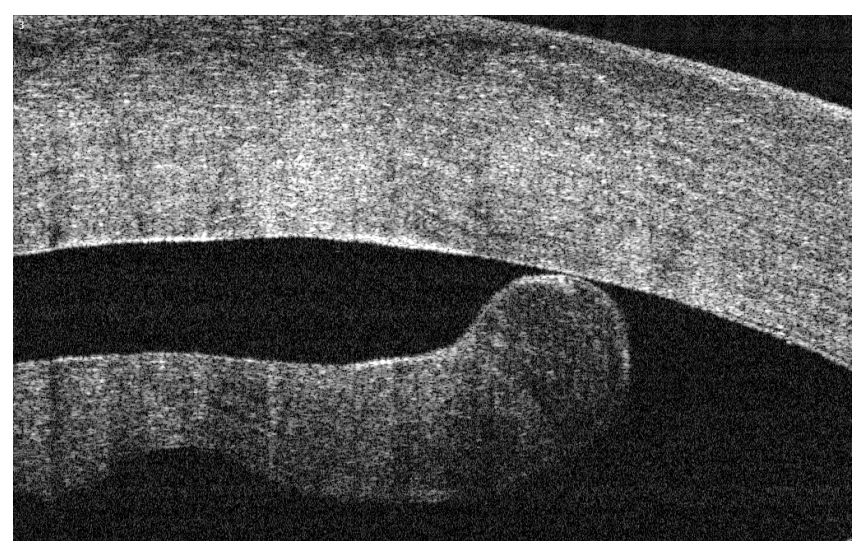

Figure 3. High-resolution OCT image of the only graft detachment in a patient with active bleb leak. Edematous lenticule with interfacial fluid is evident on this cut.

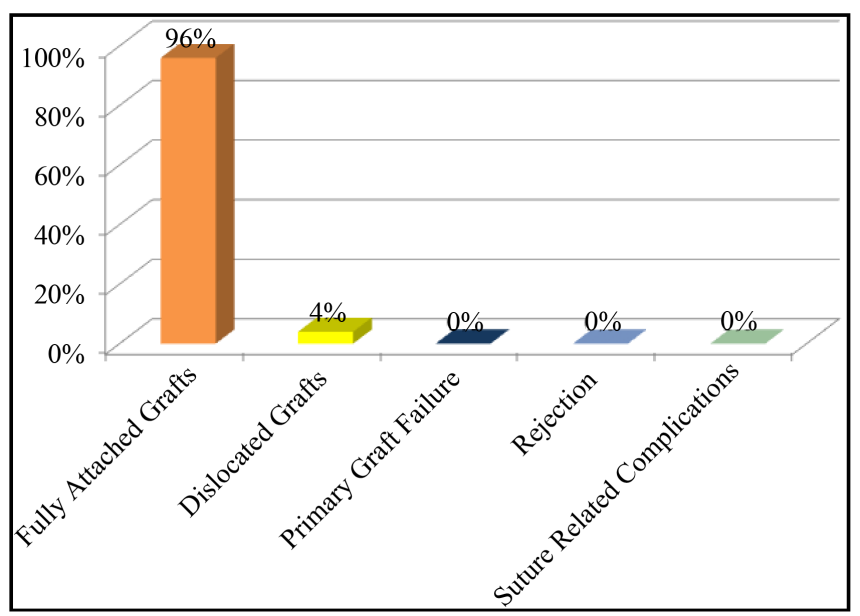

Figure 4. Graft dislocation rate was $4 \%$ and occurred in $1 / 25$ patient with an actively leaking trabeculectomy bleb. There was no incidence of primary graft failure, rejection, or suture-related complications.

\section{Discussion}

DSAEK is the preferred surgical remedy to treat endothelial failure. Despite reports of improved DSAEK techniques available in the literature, graft dislocation still represents a significant complication [5] [11]-[12]. Dislocation may be caused by remaining interface fluid, aggressive rubbing, or problems with handling, preparation, and insertion of the lenticule. Of particular concern are patients who are at high risk for detachment, including those with previous graft detachment or hypotony, those with blebs and tubes, and those with vitreous prolapse, iris abnormalities, and aphakia.

Many surgical techniques to reduce dislocation rates have been published. Price et al. [2] presented a technique involving fenestration and massaging of the cornea, which produced a dislocation rate of $6 \%$ in a report of 200 cases. Terry and colleagues reported an additional technique consisting of aggressive scraping of the peripheral recipient bed to create a rough stromal surface, as evidenced by visible stroma fibrils [3]. This technique encourages proteoglycan adherence of the graft and produced a reported 1.5\% dislocation rate. Gorovoy et al. [13] recommended the instillation of an air bubble for 1 hour to apply direct pressure of the donor lenticule to the recipient cornea. The dislocation rate with this method was $25 \%$. Lee and colleagues presented a novel technique of soaking the donor graft in an enriched balanced salt solution (BSS Plus) for 20 minutes before trephination and insertion into the anterior chamber [14]. The authors proposed that this technique reduced the lubrica- 
tion effect of the corneal storage media (Optisol GS) and facilitated easier insertion, better unfolding, and faster adherence of the graft to the recipient bed. In this series of 103 cases, a $0 \%$ dislocation rate was reported.

Other techniques to promote graft adherence had been documented as well. Meisler et al. [15] recommended the use of an air-fluid exchange system to improve control of the anterior chamber pressure and promote air tamponade the graft against the recipient cornea. Peng [16] introduced a technique combining the use of OVD and an air bubble to promote adherence in eyes with abnormal structures. More recently, Patel [17] recommended suturing the graft in one location to hold the graft in place and prevent dislocation into the posterior segment. The suture used was the same one used to drag the graft into the anterior chamber via a Busin glide. This technique relied on the gradual adherence of the remaining parts of the graft over time since there was only one point of fixation.

An ideal method to reduce detachment rates would be safe, reproducible, inexpensive, and technically simple and would avoid gross endothelial cell loss. Using a readily commercially available 10-0 nylon suture, we designed a transfixation method using three anchoring sutures that secure the lenticule to the stroma with minimal manipulation of the lenticule. The sutures in our cohort group proved useful and were routinely pulled after one week if graft adherence was confirmed. The $4 \%$ detachment rate in our case series is comparable to the rate seen with other techniques described in the literature. The one case of detachment appeared be induced by bleb leak related hypotony; this case demonstrated the importance of postoperative hypotony prevention. In cases of probable hypotony, the sutures may remain in the cornea for extended time until resolution of the hypotony.

One weakness of this transfixation technique is the suture-induced endothelial injury, which may progress to graft failure in the future. A flaw of this retrospective design is the lack of endothelial cell monitor as function of time. Future prospective investigation may elucidate the clinical relevancy of suture-associated endothelial cell loss. However, graft failure was not observed in this case series over the follow-up period, ranged from 3 to 27 months. Arguably, in patients at high risk for graft dislocation, this technique could provide a useful addition to physician's surgical repertoire.

The DSAEK procedure represents an exciting tool in the armamentarium of corneal surgeons. Despite improvements over the past several years, complications still represent a significant concern; and graft detachment is one of the leading complications. As shown in our study, Y-suture transfixation represents an effective, safe, and inexpensive means to reduce detachments, even in high-risk patients.

\section{Disclosure}

1) Financial Support: An unrestricted grant from Research to Prevent Blindness. Pho Nguyen is supported by the Heed Ophthalmic Foundation and the Fletcher Jones Foundation.

2) Financial Disclosure(s): The authors have no financial interests in the topic of this manuscript. No conflicting relationship exists for any author.

3) The views expressed in this article are those of the authors and do not necessarily reflect the official policy or position of the Department of the Navy, or Department of Defense, nor the US Government.

\section{Acknowledgements}

Thanks to Research to Prevent Blindness for their unrestricted grant which in part made this research possible.

\section{References}

[1] Eye Banking Statistical Report. Washington DC. Eye Bank Association of America; 2008.

[2] Price Jr., FW. and Price, M.O. (2006) Descemet’s Stripping with Endothelial Keratoplasty in 200 Eyes: Early Challenges and Techniques to Enhance Donor Adherence. Journal of Cataract Refractive Surgery, 32, 411-418. http://dx.doi.org/10.1016/j.jcrs.2005.12.078

[3] Terry, M., Shamie, N., Chen, E., et al. (2008) Endothelial Keratoplasty-A Simplified Technique to Minimize Graft Dislocation, Iatrogenic Graft Failure, and Pupillary Block. Ophthalmology, 115, 1179-1186. http://dx.doi.org/10.1016/j.ophtha.2007.09.005

[4] Bahar, I., Kaiserman, I., McAllum, P., et al. (2008) Comparison of Posterior Lamellar Keratoplasty Techniques to Penetrating Keratoplasty. Ophthalmology, 115, 1525-1533. http://dx.doi.org/10.1016/j.ophtha.2008.02.010

[5] Lee, W.B., Jacobs, D.S., Musch, D.C., et al. (2009) Descemet’s Stripping Endothelial Keratoplasty: Safety and Out- 
comes: A Report by the American Academy of Ophthalmology. Ophthalmology, 116, 1818-1830. http://dx.doi.org/10.1016/j.ophtha.2009.06.021

[6] Price, M.O., Gorovoy, M., Benetz, B.A., et al. (2010) Descemet’s Stripping Automated Endothelial Keratoplasty Outcomes Compared with Penetrating Keratoplasty from the Cornea Donor Study. Ophthalmology, 117, 438-444.

[7] Price, M.O. and Price, F.W. (2007) Descemet’s Stripping Endothelial Keratoplasty. Current Opinion in Ophthalmology, 18, 290-294. http://dx.doi.org/10.1097/ICU.0b013e3281a4775b

[8] Melles, G.R., Eggink, F.A., Lander, F., et al. (1998) A Surgical Technique for Posterior Lamellar Keratoplasty. Cornea, 17, 618-626. http://dx.doi.org/10.1097/00003226-199811000-00010

[9] Melles, G.R., Lander, F. and Nieuwendaal, C.P. (2002) Sutureless, Posterior Lamellar Keratoplasty: A Case Report of a Modified Technique. Cornea, 21, 325-327. http://dx.doi.org/10.1097/00003226-199811000-00010

[10] Terry, M.A. (2003) A New Approach for Endothelial Transplantation: Deep Lamellar Endothelial Keratoplasty. International Ophthalmology Clinics, 43, 183-193. http://dx.doi.org/10.1097/00004397-200343030-00017

[11] Nguyen, P., Khashabi, S., Chopra, V., Francis, B., Heur, M., Song, J.C. and Yiu, S.C. (2013) Descemet Stripping with Automated Endothelial Keratoplasty: A Comparative Study of Outcome in Patients with Preexisting Glaucoma. Saudi Journal of Ophthalmology, 27, 73-78. http://dx.doi.org/10.1016/j.sjopt.2013.02.002

[12] Singh, A., Gupta, A. and Stewart, J.M. (2010) Posterior Dislocation of Descemets Stripping Automated Endothelial Keratoplasty Graft Can Lead to Retinal Detachment. Cornea, 29, 1284-1286. http://dx.doi.org/10.1097/ICO.0b013e3181e84402

[13] Gorovoy, M.S. (2006) Descemets-Stripping Automated Endothelial Keratoplasty. Cornea, 25, 886-889. http://dx.doi.org/10.1097/01.ico.0000214224.90743.01

[14] Lee, J.K., Eghrari, A.O., Desai, N.R., Stark, W.J. and Gottsch, J.D. (2009) Presoaking Donor Corneas Reduces Graft Detachment Rates in Descemets Stripping Endothelial Keratoplasty. American Journal of Ophthalmology, 147, 439441. http://dx.doi.org/10.1016/j.ajo.2008.09.026

[15] Meisler, D.M., Dupps Jr., W.J., Covert, D.J. and Koenig, S.B. (2007) Use of an Air-Fluid Exchange System to Promote Graft Adhesion during Descemet's Stripping Automated Endothelial Keratoplasty. Journal of Cataract \& Refractive Surgery, 33, 770-772. http://dx.doi.org/10.1016/j.jcrs.2006.11.033

[16] Peng, R., Hao, Y.S., Chen, H.J., Sun, Y. and Hong, J. (2009) Endothelial Keratoplasty: The Use of Viscoelastic as an Aid in Reattaching the Dislocated Graft in Abnormally Structured Eyes. Ophthalmology, 116, 1897-1900. http://dx.doi.org/10.1016/j.ophtha.2009.03.049

[17] Patel, A.K., Luccarelli, S., Ponzin, D. and Busin, M. (2011) Transcorneal Suture Fixation of Posterior Lamellar Grafts in Eyes with Minimum or Absent Iris-Lens Diaphragm. American Journal of Ophthalmology, 151, 460-464. http://dx.doi.org/10.1016/j.ajo.2010.08.043 
Scientific Research Publishing (SCIRP) is one of the largest Open Access journal publishers. It is currently publishing more than 200 open access, online, peer-reviewed journals covering a wide range of academic disciplines. SCIRP serves the worldwide academic communities and contributes to the progress and application of science with its publication.

Other selected journals from SCIRP are listed as below. Submit your manuscript to us via either submit@scirp.org or Online Submission Portal.
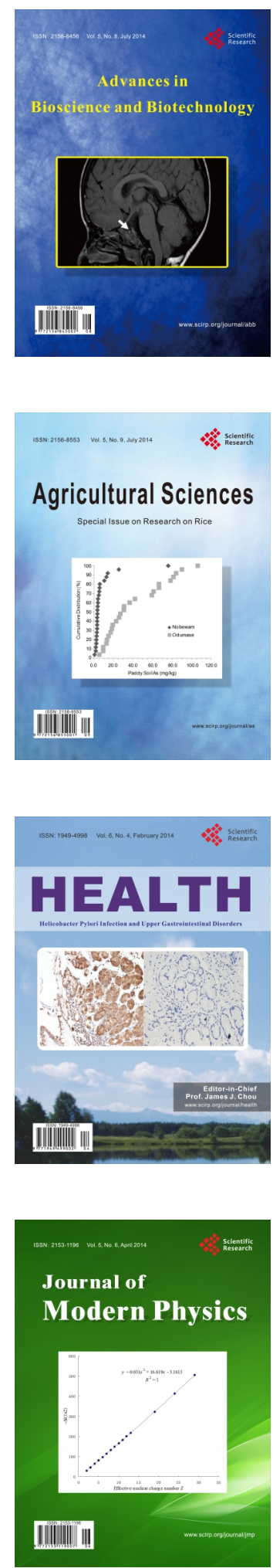
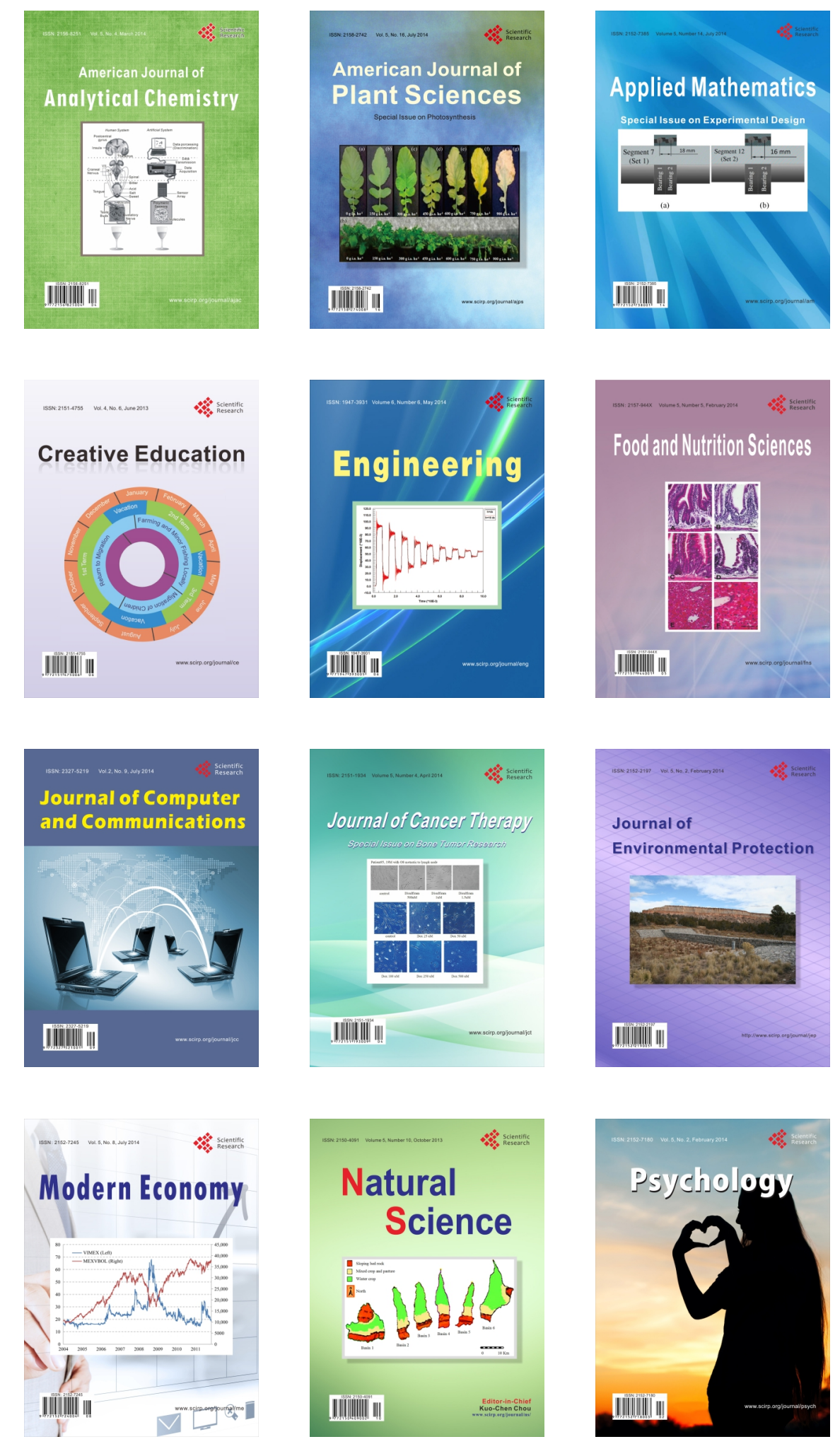